\title{
Zum Hören von Stechmücken
}

\author{
Von Erhard Keppler * \\ Aus dem Institut für Schwingungsforschung in Tübingen \\ (Z. Naturforschg. 13 b, 285-286 [1958]; eingegangen am 18. Februar 1958)
}

\begin{abstract}
Es soll hier über Versuche berichtet werden, die im Anschluß an Untersuchungen über das Richtungshören von Stechmücken, über die früher ${ }^{1}$ berichtet wurde, zur Ausführung gelangten. Es handelt sich dabei zum Teil um Wiederholungen zuerst von Rотн ${ }^{2}$ ausgeführter Versuche, wobei aber hier abweichend von Rотн, der das Verhalten der Tiere nach Ausführung gewisser Operationen beobachtete, die Auswirkung dieser Operationen auf elektrophysiologischem Wege messend verfolgt wurde. Die Schlüsse, die aus diesen Messungen zu ziehen sind - entsprechend den in l.c. ${ }^{1}$ entwickelten Vorstellungen - bestätigten Rотнs Beobachtungen.
\end{abstract}

Männliche Stechmücken (Aedes aegypti), an deren Antennen die nachstehend beschriebenen Operationen ausgeführt worden waren, wurden in ein von einem Lautsprecher erzeugtes Schallfeld von sinusförmigem Verlauf gebracht, dessen Frequenz mit dem Grundton des von weiblichen Tieren abgestrahlten Fluggeräusches (etwa $300 \mathrm{~Hz}$ bei Zimmertemperatur) ${ }^{3}$ übereinstimmte. An den Tieren konnte mit der in 1. c. ${ }^{1}$ beschriebenen Versuchsanordnung durch Einführung von zwei Elektroden (in den basalen Teil des Pedicellus und in den Thorax) elektrische Spannungen („Schallspannungen“ nach einem Vorschlag von Tischner ${ }^{4}$ ) von der Größenordnung einiger $50 \mu \mathrm{V}$ abgeleitet werden. Aus dem Verlauf dieser Spannungen kann man, im Anschluß an die Untersuchungen in l. c. ${ }^{1}$ Aussagen über das Hören der Tiere machen.

\section{Ergebnisse}

a) Entfernt man die Flagellarsegmente einer Antenne dicht über dem Pedicellus und legt man den verbleibenden Stummel geeignet (z. B. mit Schellack) fest, oder wird die Bewegung des Flagellum relativ zum Pedicellus durch entsprechende Maßnahmen verhindert, so treten im Schallfeld beliebiger Frequenz keine Schallspannungen auf. Die Tiere sind in diesem Zustand also akustisch unempfindlich. Dies fand auch Rотн.

b) Die Frequenz, bei der im Schallfeld das Maximum der Schallspannung bei unversehrter Antenne eintritt, stimmt überein mit der mechanischen Re. sonanzfrequenz der Antenne (Abb. 1, Kurve a). Wird die Spitze der Antenne mit einem Schellacktropfen beschwert, so erniedrigt sich die Frequenz, bei der die Schallspannung ihr Maximum annimmt (entsprechend einer Erniedrigung der Resonanz-

1 E. Keppler, Z. Naturforschg. 13 b, 280 [1958].

2 L. M. Rотн, Amer. Naturalist 40, 265 [1948].

3 H. Tischner u. A. Schief, Verh. dtsch. zool. Ges. Tübingen, 51, 453 [1954]. frequenz der Antenne), (Abb. 1, Kurve b), die Schallspannung wird kleiner; die Tiere sind also weniger empfindlich gegen Schall. Dasselbe konstatiert auch Rотн. Entfernt man die beschwerte Spitze, so erhöht sich die Maximum-Frequenz geringfügig; die Schallspannung wird größer (Abb. 1, Kurve c).

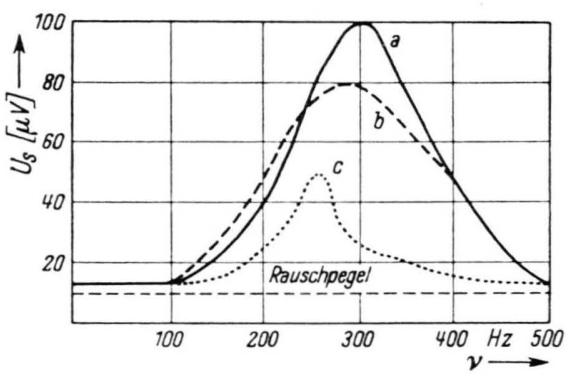

Abb. 1. Schallspannung $U_{S}$ in Funktion der Frequenz $v$.

Die Tiere reagieren wieder eher auf akustische Reizung, was auch Rотн fand. Es kommt also darauf an, daß die Antenne genügend viel Schallenergie aufnimmt, d. h. mit genügend großer Amplitude schwingt. Da sie im zweiten Fall außerhalb der Resonanz angeregt wird (konstante erregende Frequenz), ist dies verständlich.

c) Abb. 2, Kurve a, zeigt den Frequenzverlauf der Schallspannung bei unversehrter Antenne, Kurve b. nachdem ein Viertel, Kurve c, nachdem die Hälfte des Flagellum abgeschnitten wurde. Mit kürzer werdender Antenne verkleinert sich die Schallspannung (Verringerung der Oberfläche, in zweiter Linie auch physiologische Schädigung; letztere beeinflußt das grundsätzliche Ergebnis nicht. Vgl. hierzu 1. c. ${ }^{5}$ ). Be-

${ }^{4}$ H. Tischner, Acustica [Zürich] 3, 335 [1953]:

* Anschrift des Verfassers: Max-Planck-Institut für Aeronomie, Lindau (Harz).

5 U. Bässler, Naturwissenschaften 44, 336 [1957]. 
merkenswerterweise erniedrigt sich auch die Frequenz. an der die jeweiligen Maxima der Schallspannung auftreten, obwohl man rein mechanisch eine Erhöhung der Resonanzfrequenz erwarten würde. Hierïur ist der Druckverlust, den der durch die Operation ausgelöste Blutverlust hervorruft, verantwortlich (Verminderung der Einspannung der Basalplatte des Flagellum im Pedicellus ${ }^{6}$ ).

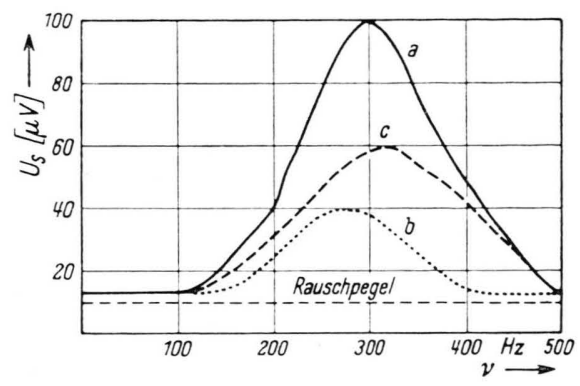

Abb. 2. Schallspannung $U_{s}$ in Funktion der Frequenz $v$. d) Der Frequenzbereich, in dem die Tiere hören können, ergibt sich aus den Messungen im Schallfeld entsprechend dem früheren Befund ${ }^{1}$ zu 100 bis $500 \mathrm{~Hz}$. (Rотн gab als obere Grenze $750 \mathrm{~Hz}$ an, was nach meinen Messungen zu hoch ist.)

e) Durch Vergleich mit dem früheren Ergebnis ${ }^{1}$. wonach bei mechanischer Erregung die Schallspannungs-Amplitude bei konstanter Antennenelongation unabhängig von der Frequenz ist, ergibt sich, daß der charakteristische Frequenzgang der Schallspannungs-Amplitude (Abb. 2, Kurve a) allein bedingt ist durch das Resonanzverhalten der Antenne, daß das Johnston-Organ selbst zwischen 100 und $500 \mathrm{~Hz}$ frequenzunabhängig arbeitet.

Die Untersuchungen wurden im Institut für Schwingungsforschung, Tübingen, durchgeführt. Ich danke Herrn Prof. Dr. H. Tischner für die Bereitstellung der Mittel sowie für zahlreiche, anregende Diskussionen.

\title{
Über die Verteilung von Radioyttrium und radioaktiven Seltenen Erden im Säugerorganismus
}

\author{
I.: Verteilungsversuche mit trägerfreiem Radioyttrium $\left({ }^{91} \mathrm{Y}\right)$ an Meerschweinchen \\ Von Ernst Spode
}

\begin{abstract}
Aus dem Institut für Medizin und Biologie der Deutschen Akademie der Wissenschaften zu BerlinBuch (Präsident: Prof. Dr. Dr. h. c. W. FrIedrich), Arbeitsbereich Angewandte Isotopenforschung (Z. Naturforschg. 13 b, 286-291 [1958] ; eingegangen am 27. Dezember 1957)
\end{abstract}

\begin{abstract}
Es wird über das Verteilungsmuster von Radioyttrium $\left.{ }^{91} \mathrm{Y}\right)$ in Meerschweinchen nach intrakardialer, intraperitonealer und subkutaner Applikationsweise und die dabei auftretenden Unterschiede berichtet. Nach der Inkorporation von Yttrium muß mit einer Leberpassage vor der Knochenspeicherung gerechnet werden. Die hohen Aktivitäten in Leber und Nieren bedürfen mehr als bisher der strahlenbiologischen Beachtung.
\end{abstract}

Das Interesse für die radioaktiven Yttriumisotope ${ }^{90} \mathrm{Y}$ und ${ }^{91} \mathrm{Y}$ ist im Laufe der Zeit ständig gestiegen. Die Gründe hierfür liegen einmal in der Tatsache. daß ${ }^{90} \mathrm{Y}$, Folgeprodukt von ${ }^{90} \mathrm{Sr}$, in der Therapie eine gewisse Rolle zu spielen beginnt, teils als ge. schlossenes Präparat zur Kontaktbestrahlung, teils in kolloidaler Form für intrakavitäre Anwendung. Zum anderen tritt ${ }^{91} \mathrm{Y}$ als Spaltprodukt bei der Kern-

1 E. L. Dobson, J. W. Gofmax, H. B. Jones, L. S. Kelly u. I. A. Walker, J. Labor. clin. Med. 34, 305 [1949].

2 J. Schubert, J. Labor. clin. Med. 34, 313 [1949].

3 J. Schubert u. M. R. White, J. biol. Chemistry 184, 191 [1950].

4 J. Schubert, M. P. Finkel, M. R. White U. G. M. Hirsch, J. biol. Chemistry 182, 635 [1950].

5 B. P. Kidman, M. L. Tutt u. J. M. Vaughan, a) J. Pathol. Bacteriol. 62, 209 [1950]; b) J. Pathol. Bacteriol. 63, 253 [1951]; c) Nature [London] 167, 858 [1951]. spaltung in größerer Menge auf und muß wegen seiner Langlebigkeit und seines biologischen Verhaltens zu den besonders gefährlichen Radioisotopen gerechnet werden. Untersuchungen über die Verteilung des Radioyttriums im Versuchstier sind daher mit beiden Isotopen schon mehrfach durchgeführt worden ${ }^{1-8}$. Allerdings wurden dabei meist nur die wesentlichsten Organe (z. B. Leber, Milz, Knochen)

\footnotetext{
6 N. S. McDonald, R. E. Nusbaum, G. V. Alexander. F. Ezirliam, P. Spain u. D. E. Rounds, J. biol. Chemistry 195, 837 [1952].

7 R. Lewin. H. E. Hart, J. Greenberg, H. Spencer, K. G. Stern u. D. Laszlo, a) Proc. Radioisotope Conf. 1, 125 [1954]; b) Science [Washington] 119, 880 [1954].

8 G. A. Andrews, G. C. Kyker, R. M. Kniseley u. E. L. Palmer, Proc. Amer. Assoc. Cancer Res. 2, 46 [1955].
} 